УДК 544.65:544.4+544.653.23:547.1-32-304.2

DOI: $10.17308 / \mathrm{kcmf} .2019 .21 / 718$

Поступила в редакцию 19.12.2018

Подписана в печать 15.02.2019

\title{
РОЛЬ СТРУКТУРНО-МОРФОЛОГИЧЕСКОГО СОСТОЯНИЯ ПОВЕРХНОСТИ ПЛАТИНЫ В КИНЕТИЧЕСКИХ И ТЕРМОДИНАМИЧЕСКИХ ХАРАКТЕРИСТИКАХ ПРОЦЕССА АДСОРБЦИИ АНИОНА СЕРИНА
}

\author{
(ㄱ 2019 Н. Е. Кулешова, Е. В. Бобринская, А. В. Введенский*, Е. Б. Рычкова \\ Воронежский государственный университет \\ Университетская пл., 1, 394018 Воронеж, Россия
}

\begin{abstract}
Аннотация. Исследована адсорбция аниона серина на гладком Pt и $\mathrm{Pt}(\mathrm{Pt})$-электроде. Методом кривых заряжения получены стационарные и кинетические изотермы адсорбции. Установлено, что как на гладком, так и $\mathrm{Pt}(\mathrm{Pt})$-электроде, кинетика исследуемых процессов подчиняется уравнению Рогинского-Зельдовича, а стационарное заполнение описывается изотермой Темкина. При этом адсорбция аниона серина на $\mathrm{Pt}(\mathrm{Pt})$ сопровождается диссоциацией адсорбата. Найдены основные термодинамические характеристики (константа адсорбционного и изменение свободной энергии Гиббса) процесса адсорбции аниона серина на обоих электродах.
\end{abstract}

Ключевые слова: серин, аминокислота, платина, адсорбция, изотерма.

\section{ВВЕДЕНИЕ}

Адсорбция органических веществ в ходе электродного процесса может рассматриваться как элементарная стадия [1-4], а, следовательно, установление закономерностей адсорбции и природы адсорбата необходимо для понимания механизма электрохимической реакции. Поскольку адсорбционный процесс наряду с вытеснением молекул растворителя с занимаемых адсорбционных центров может характеризоваться еще и достаточно сильным взаимодействием атомов металла с частицами адсорбата, то в ходе адсорбционного накопления последний может подвергаться деструкции $[1,3,5-7]$.

Таким образом, формально-кинетическое описание электродной реакции, сопровождающейся адсорбцией реагентов, продуктов или интермедиатов, требует конкретизации элементарного акта адсорбционного процесса. Это означает, что установлен диссоциативный или недиссоциативный характер адсорбции, определено количество активных центров поверхности, захватываемых частицей адсорбата, а кроме того - выявлен тип адсорбционной изотермы, что в значительной мере также обусловлено состоянием поверхности адсорбента и спецификой межчастичных взаимодействий.

$\checkmark$ Введенский Александр Викторович, e-mail: alvved@chem.vsu.ru
Лишь в некоторых случаях, подобные данные могут быть получены при помощи отражательной in situ ИК - спектроскопии $[8,9]$, импедансометрии [10], методом радиоактивных индикаторов [11], либо найдены в результате квантово-химических расчетов [12-13]. Установлено, в частности, что глицин [14-21], $\alpha$-аланин [23, 24, 27], а также серин [22-27] в процессе жидкофазной адсорбции подвергаются деструкции на поверхности платины. Имеющиеся данные указывают, что состав и свойства адсорбированных частиц зависят от многих факторов, в том числе от строения молекулы аминокислоты, степени упорядоченности поверхности электрода (в частности, степени ее развития), потенциала и кислотности среды. Однако в исследованиях, зачастую, констатируется только сам факт адсорбции, реже приводятся изотермы адсорбции аминокислот, в частности серина. Кроме того, нами ранее [28] установлено, что скорость процесса электроокисления аниона серина на платинированном платиновом электроде существенно превышает таковую для компактного платинового электрода. Причем данное отличие не связано с увеличением площади поверхности платины.

В связи с этим, цель настоящей работы - установление влияния различий в структурно-морфологическом состоянии поверхности $\mathrm{Pt}$ и $\mathrm{Pt}(\mathrm{Pt})$ электродов (прежде всего - степени ее развития) 
на основные закономерности и термодинамические характеристики процесса адсорбции аниона серина.

\section{ЭКСПЕРИМЕНТАЛЬНАЯ ЧАСТЬ}

Измерения проведены в электрохимической ячейке с неразделенным катодным и анодным пространствами в атмосфере х.ч. аргона методом электроокисления в адсорбированном слое (кривых заряжения) [1, 29-30] с помощью компьютеризированного комплекса IPC-Compact. В качестве рабочих электродов использовали платинированную и не платинированную платиновую сетку. Истинную площадь поверхности соответствующего рабочего электрода определяли по заряду десорбции атомарного водорода также методом кривых заряжения [31-33]. Зная геометрическую и истинную площадь поверхности платинового электрода, оценили значение фактора шероховатости поверхности. Для гладкого платинового электрода его величина составила $3.0 \pm 0.35$; для платинированного $100 \pm 12$. Значения факторов шероховатости использовали при расчете плотности тока для получения кривых заряжения.

Насыщенный хлоридсеребряный электрод сравнения отделялся от рабочего пространства ячейки капилляром Луггина, вспомогательным электродом служила платиновая проволока. Потенциалы в работе приведены относительно потенциала стандартного водородного электрода (с.в.э.), $T \sim 298$ К. Перед измерениями рабочий электрод подвергали анодно-катодной активации в фоновом $0.1 \mathrm{M}$ растворе $\mathrm{NaOH}$ (при потенциалах $E=1.20$ и $-0.80 \mathrm{~V}$ соответственно) для удаления возможных электроактивных примесей.

Растворы D,L-серина $\left(2.0 \cdot 10^{-3} \div 5.0 \cdot 10^{-2} \mathrm{~mol} / \mathrm{dm}^{3}\right)$ готовили по навеске вещества (ч.д.а.) в $0.1 \mathrm{M}$ растворе гидроксида натрия (х.ч.) на бидистилляте. Согласно соотношению между $\mathrm{pH}$ и $\mathrm{pK}$ дис карбоксильной и аминогрупп, аминокислота в растворе преимущественно существует в виде аниона.

Потенциал адсорбции $\left(E_{\text {адс }}\right)$ выбирали по данным линейной вольтамперометрии [31], критерием выбора служило отсутствие фарадеевских процессов окисления/восстановления аниона аминокислоты. Таким образом, для гладкого и платинированного платинового электрода значения $E_{\text {адс }}=-0.05$ и $-0.10 \mathrm{~V}$ соответственно. После активации поверхности платинового электрода в ячейку вводили аликвоту щелочного раствора аминокислоты и выдерживали, при данном значении $E_{\text {адс }}$, определенное время $t_{\text {адс }}$. Затем меняли режим поляризации на гальваностатический и подавали на электрод анодный ток, отвечающий началу процесса электроокисления аминокислоты. Аналогичным образом, но, не вводя аминокислоту в раствор, получали кривую заряжения в фоновом электролите.

\section{РЕЗУЛЬТАТЫ И ОБСУЖДЕНИЕ}

Типичные кривые заряжения Pt-электрода в растворе фона, а также после накопления на его поверхности при $E_{\text {адс }}=-0.05 \mathrm{~V}$ слоя адсорбированных анионов серина представлены на рис. 1. С ростом фактора шероховатости поверхности при переходе от гладкого к платинированному платиновому электроду в фоновом растворе происходит существенное увеличение области потенциалов десорбции атомарного водорода. Внесение аминокислоты приводит к изменению общего вида $E, t$-зависимости. Так, начиная с $E \sim 0.20 \mathrm{~V}$, на кривой заряжения в растворе, содержащем Ser-, возникает излом, отвечающий протеканию процесса анодного окисления аниона аминокислоты. При потенциале $\sim 0.75 \mathrm{~V}$ все $E$, $t$-кривые совпадают, что свидетельствует о полном окислении органического вещества до продуктов, практически не адсорбирующихся на электроде.

Установлено, что увеличение времени адсорбции и концентрации серина в растворе приводит к росту анодного заряда, затраченного на электроокисление адсорбированных анионов Ser- на электроде. Поскольку область потенциалов десорбции ад-атомов водорода на компактном платиновом электроде крайне мала и плохо воспроизводится, то степень заполнения поверхности платины анионом серина рассчитывали по формуле:

$$
\Theta_{\text {Ser }}=\frac{q_{\mathrm{O}}^{\Phi}-q_{\mathrm{O}}}{q_{\mathrm{O}}^{\Phi}} .
$$

Здесь $q^{\phi}-$ заряд, затраченный на создание монослоя атомарного кислорода $(\mathrm{O})$ на электроде в растворе фона, $q$ - аналогичный заряд, найденный по кривой заряжения после выдержки электрода при заданном значении $E_{a д c}$ в растворе с добавкой аминокислоты. Полученные таким образом кинетические изотермы адсорбции аниона серина на $\mathrm{Pt}$ и $\mathrm{Pt}(\mathrm{Pt})$ электродах, перестроенные в полулогарифмических координатах, представлены на рис. 2.

На кинетических изотермах адсорбции Ser- на $\mathrm{Pt}(\mathrm{Pt})$ фактически отсутствует индукционный период, а увеличение концентрации аминокислоты в растворе приводит к увеличению степени заполнения поверхности. В то же время в случае адсорбции Ser- на гладкой платине при $c_{\mathrm{Ser}^{-}} \leq 0.01 \mathrm{M}$ на 

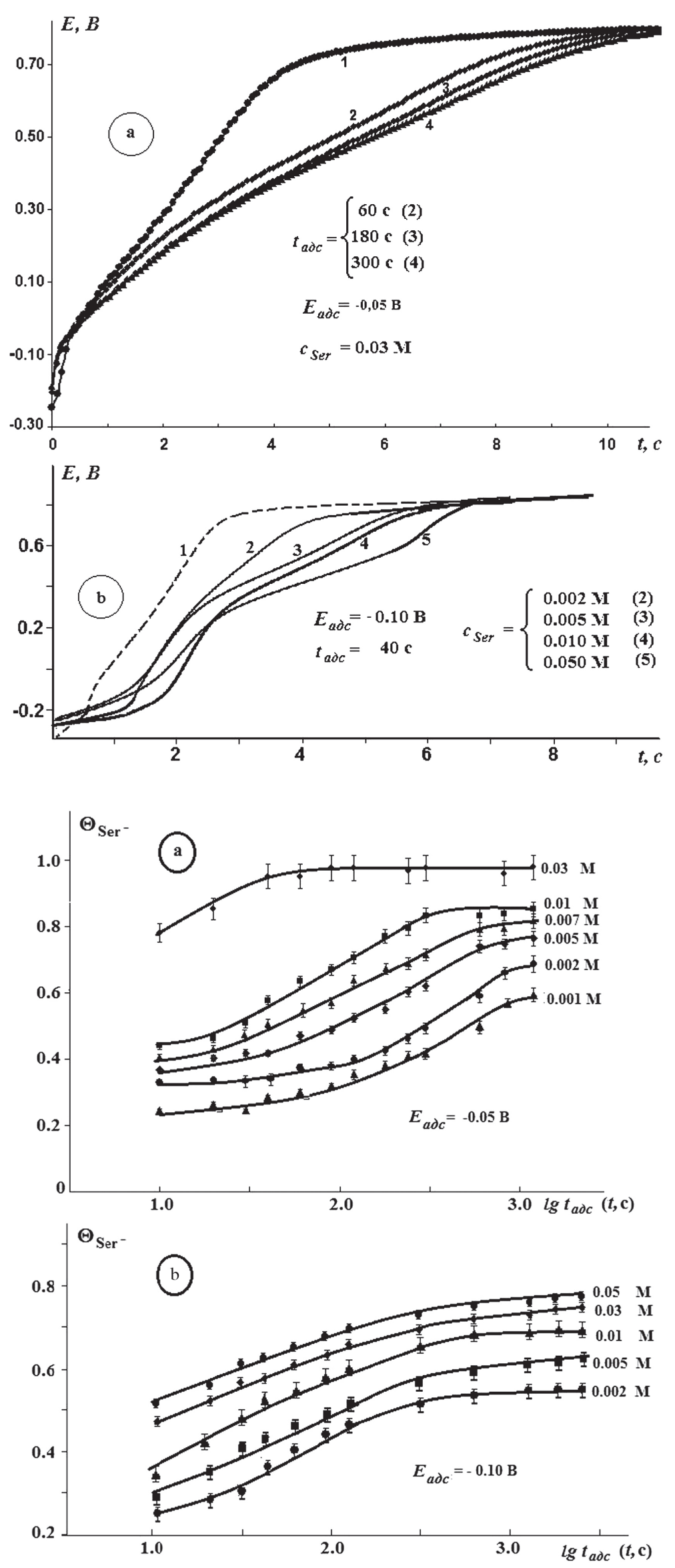

Рис. 1. Кривые заряжения, полученные на гладком $(a)$ и $\mathrm{Pt}(\mathrm{Pt})$-электроде $(b)$ при $i=0.5 \mathrm{~mA} / \mathrm{cm}^{2}$ в растворе $0.1 \mathrm{M} \mathrm{NaOH} \mathrm{(1)} \mathrm{и}$ с добавкой Ser после выдержки при $E_{\text {адс }}$

[Fig. 1. Charging curves obtained on a smooth (a) and $\mathrm{Pt}(\mathrm{Pt})$ electrode $(b)$ at $i=0.5 \mathrm{~mA} / \mathrm{cm}^{2}$ in $0.1 \mathrm{M} \mathrm{NaOH}$ (1) solution and with the addition of Ser after exposure at $E_{\text {ads }}$

Рис. 2. Кинетические изотермы адсорбции аниона серина на гладком $(a)$ и платинированном платиновом электроде $(b)$

[Fig. 2. Kinetic isotherms of adsorption of serine anion on smooth $(a)$ and platinized platinum electrode $(b)]$ 
кинетических изотермах адсорбции появляется индукционный период, продолжительность которого, как и время достижения стационарного заполнения поверхности электрода уменьшается с ростом концентрации аминокислоты. Стоит заметить, что значения степени заполнения поверхности для гладкого Pt-электрода выше, при одной и той же объемной концентрации Ser, чем для платинированного. Скорее всего, это связано с тем, что более развитая поверхность $\mathrm{Pt}(\mathrm{Pt})$ характеризуется также и большей энергетической неоднородностью. В области средних заполнений на обеих $\Theta_{\mathrm{Ser}}, \lg t_{04 \mathrm{~A}^{-}}$зависимостях удается выделить достаточно протяженные линейные участки, наклон которых практически не зависит от объемной концентрации серина.

На следующем этапе путем сопоставления экспериментально полученных стационарных и кине-
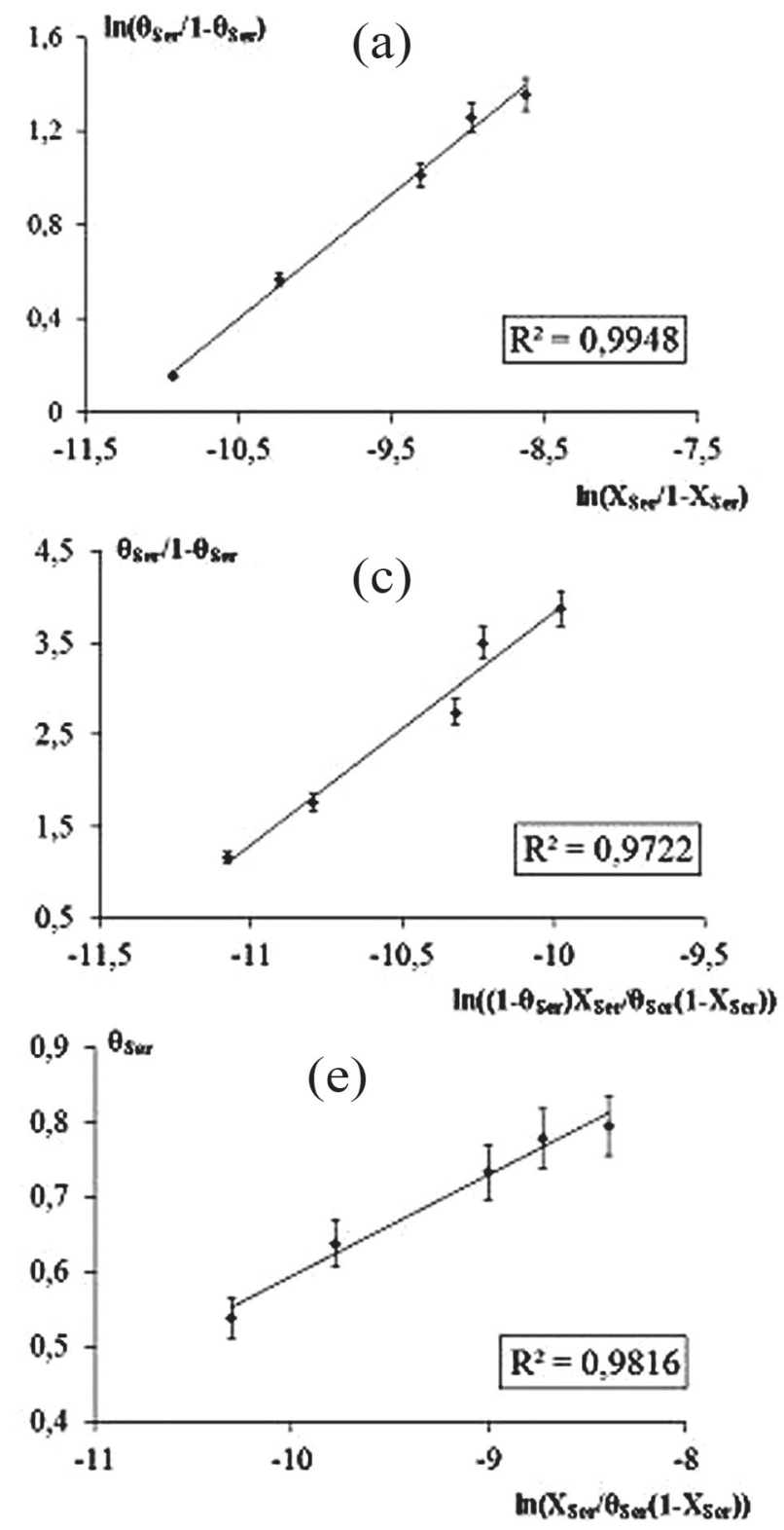

тических изотерм с имеющейся базой теоретически найденных [34], устанавливали тип изотермы, наилучшим образом описывающей процесс адсорбции серина. Математический анализ проводили в два этапа. На первом - экспериментальные $\Theta_{\text {Ser }}, c_{\text {Ser }}{ }^{-}$ зависимости перестраивали в полулогарифмических координатах, соответствующих базе основных изотерм одноцентровой адсорбции [34].

Анализ показал, что в этих координатах большинство из использованных изотерм в принципе может быть привлечено для описания исследуемого адсорбционного процесса. Действительно, в соответствующих критериальных координатах экспериментальные стационарные изотермы оказываются линейными, причем с достаточно высокими коэффициентами корреляции (рис. 3-4). В силу этого, однозначный вывод о типе адсорбционной изотер-
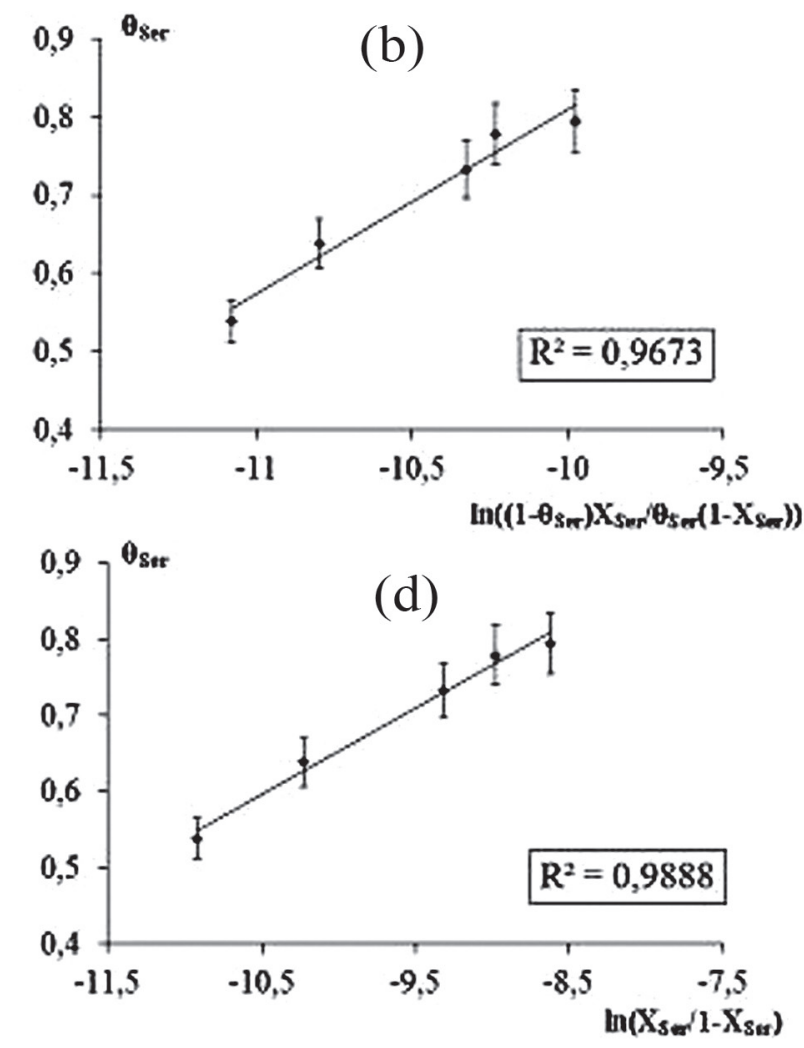

Рис. 3. Концентрационные изотермы адсорбции серина на Pt электроде, представленные в полулогарифмических или двойных логарифмических координатах: $a$ ) изотерма Лэнгмюра; $b$ ) изотерма Фрумкина;

c) изотерма Фольмера; $d$ ) изотерма Темкина; $e$ ) вириальная изотерма

[Fig. 3. Concentration adsorption isotherms of serine on Pt electrode, presented in semi-logarithmic or double logarithmic coordinates: a) Langmuir's isotherm;

b) Frumkin's isotherm; c) Folmer's isotherm; $d$ ) Temkin's isotherm; $e$ ) virial isotherm] 

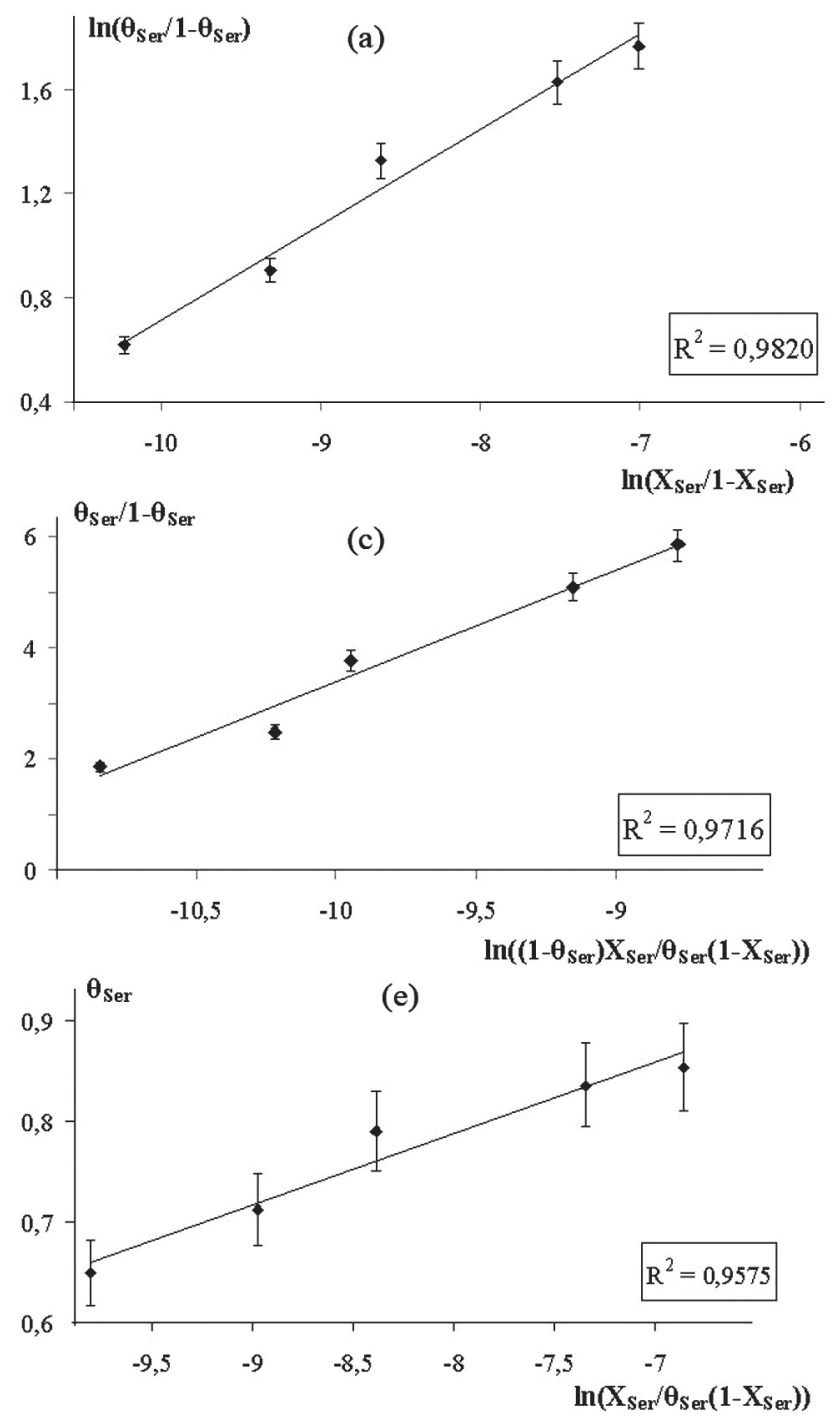

мы, а, следовательно, и характере адсорбции аниона Ser- на платине, невозможен. Поэтому на втором этапе обработки опытная $\Theta_{\mathrm{Ser}}, c_{\mathrm{Ser}}$-кривая перестраивалась в т. н. «прямых» координатах, индивидуальных для каждого типа изотермы, причем дополнительно учитывающих возможность диссоциации адсорбата. При этом параметры, необходимые для такого перестроения, оценивали из наклонов соответствующей изотермы, предварительно линеаризованной в логарифмических координатах.

Оказалось, что адсорбция Ser- на гладком Ptэлектроде удовлетворительно описывается как простой вириальной изотермой, так и изотермой Темкина [34] в отсутствии диссоциации адсорбата:

$$
e^{f \Theta_{\mathrm{Ser}}}=K_{a}(0) \frac{X_{\mathrm{Ser}}}{1-X_{\mathrm{Ser}}} .
$$

Учитывая, что параметры, которые можно оценить, используя вириальную изотерму, не имеют
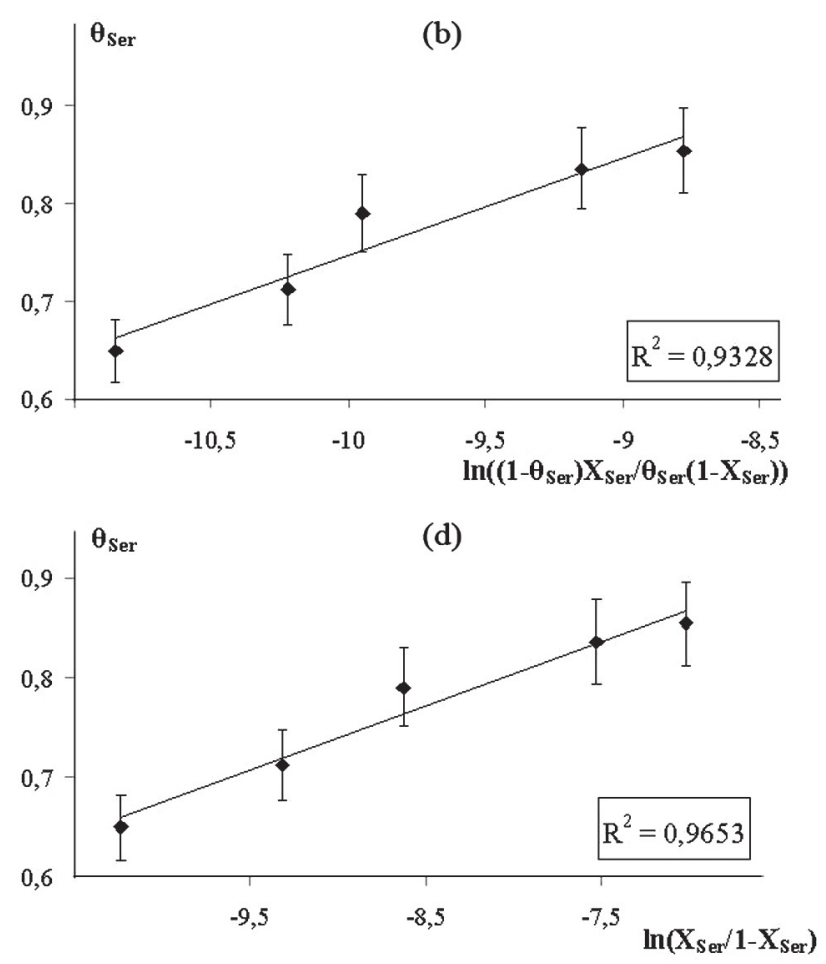

Рис. 4. Концентрационные изотермы адсорбции серина на $\mathrm{Pt}(\mathrm{Pt})$ электроде, представленные в полулогарифмических или двойных логарифмических координатах: $a$ ) изотерма Лэнгмюра; $b$ ) изотерма Фрумкина; $c$ ) изотерма Фольмера; $d$ ) изотерма Темкина; $е$ ) вириальная изотерма

[Fig. 4. Concentration adsorption isotherms of serine on $\mathrm{Pt}(\mathrm{Pt})$ electrode, presented in semi-logarithmic or double logarithmic coordinates: $a$ ) Langmuir's isotherm; $b$ ) Frumkin's isotherm; c) Folmer's isotherm; $d$ ) Temkin's isotherm; e) virial isotherm]

четкого физического смысла, далее, для определенности, будем считать, что адсорбция аниона серина на гладкой платине является недиссоциативной и описывается изотермой Темкина (рис. 5), т. е. протекает на энергетически равномерно-неоднородной поверхности.

В отличие от гладкой $\mathrm{Pt}$, на $\mathrm{Pt}(\mathrm{Pt})$-электроде опытные изотермы остаются нелинейными, если описании процесса адсорбции серина не учитывать диссоциацию адсорбата. Поэтому для описания процесса адсорбции серина на платинированном электроде $\Theta_{\mathrm{Ser}}, c_{\mathrm{Ser}}$-зависимости перестраивались в координатах, учитывающих эффект диссоциации [34]. Оказалось, что при этом только две из используемых изотерм, а именно изотермы Фрумкина и Тёмкина, адекватно описывают исследуемый адсорбционный процесс (рис. 6).

Естественно, полученные данные не позволяют установить, проявляется ли при адсорбции анио- 
на серина биографическая энергетическая неоднородность поверхности платины (модель Темкина), или доминирует индуцированная неоднородность (модель Фрумкина); в первом случае определяющими являются взаимодействия $\mathrm{Pt}-\mathrm{Ser}_{\text {адс }}^{-}$, во втоpoм $\mathrm{Ser}_{\text {адс }}^{-}-\mathrm{Ser}_{\text {адс }}^{-}$.

Отметим, что вывод о диссоциации анионов серина в ходе адсорбции на $\mathrm{Pt}(\mathrm{Pt})$ согласуется с представленным нами в [31] механизмом процесса электроокисления Ser, на основании данных линейной вольтамперометрии и кулонометрии.

Для дальнейшего анализа и сравнения адсорбционных процессов, протекающих на электродах с различным морфологическим состоянием поверхности, и расчета их количественных характеристик выбрана изотерма Темкина.

Процесс адсорбции аниона серина из водного раствора носит, несомненно, обменный характер и, в общем, может быть представлен так:

$$
\operatorname{Ser}^{-}+\mathrm{H}_{2} \mathrm{O}_{(a)} \underset{k_{2}}{\stackrel{k_{1}}{\Leftrightarrow}} \operatorname{Ser}_{(a)}^{-}+\mathrm{H}_{2} \mathrm{O} \text {. }
$$

Здесь (а) - адсорбционный центр на поверхности платины изначально занятый молекулой растворителя (воды); $k_{1}$ и $k_{2}-$ константы скорости адсорбции и десорбции соответственно. Если поверхность электрода энергетически равномерно-неоднородна, в области средних заполнений справедлива логарифмическая изотерма адсорбции Темкина, то кинетика этого процесса подчиняется уравнению Рогинского-Зельдовича [1]:

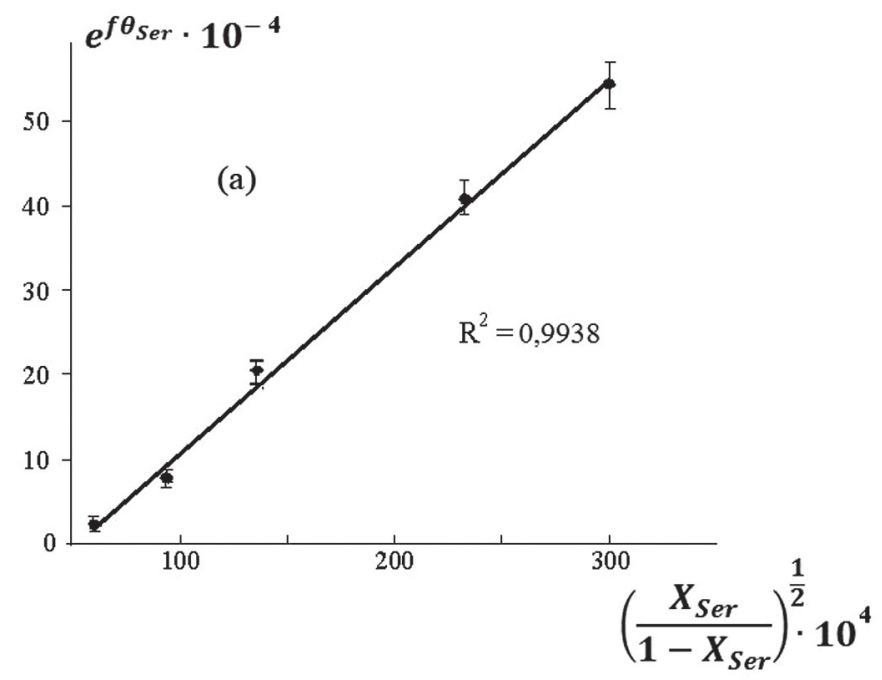

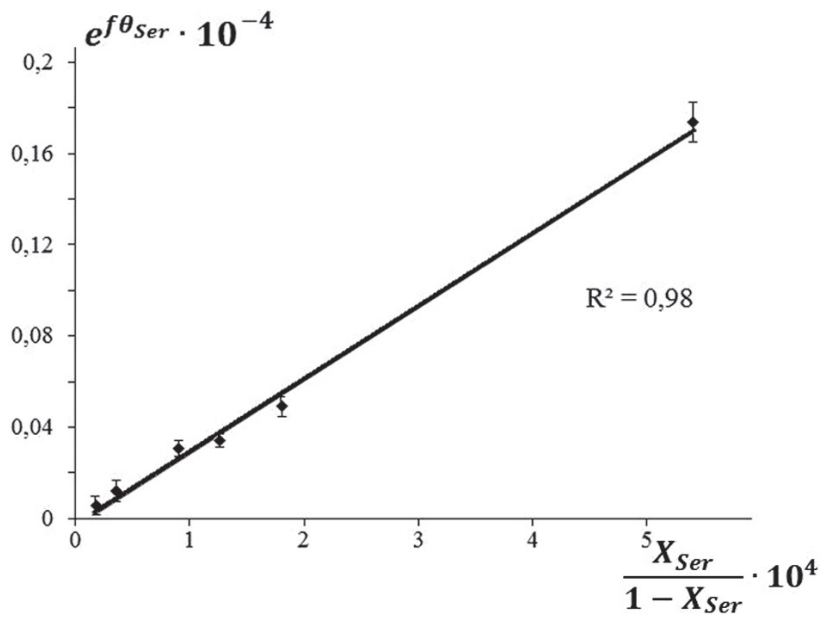

Рис. 5. Квазистационарная изотерма Темкина без учета эффекта диссоциации адсорбата, полученная для процесса адсорбции аниона серина на гладкой $\mathrm{Pt}$

[Fig. 5. Quasi-stationary Temkin's isotherm without taking into account the dissociation effect of adsorbate obtained for the adsorption of serine anion on a smooth Pt]

$$
\Theta_{\mathrm{Ser}^{-}}=\frac{1}{\alpha \bar{f}} \ln \left(\alpha \bar{f} \cdot k_{1}^{0} \frac{X_{\mathrm{Ser}^{-}}}{1-X_{\mathrm{Ser}^{-}}}\right)+\frac{1}{\alpha \bar{f}} \ln t .
$$

Здесь $\alpha$ - коэффициент симметрии потенциального барьера процесса адсорбции, $k_{1}^{0}-$ константа скорости адсорбции на участках с максимальной энергией при $\Theta_{\mathrm{Ser}} \rightarrow 0$. Отметим, что фактор энергетической неоднородности представляет собой разность $\bar{f}=f_{\mathrm{Ser}}-f_{\mathrm{H}_{2} \mathrm{O}}$, где факторы неоднородности поверхности применительно к адсорбции

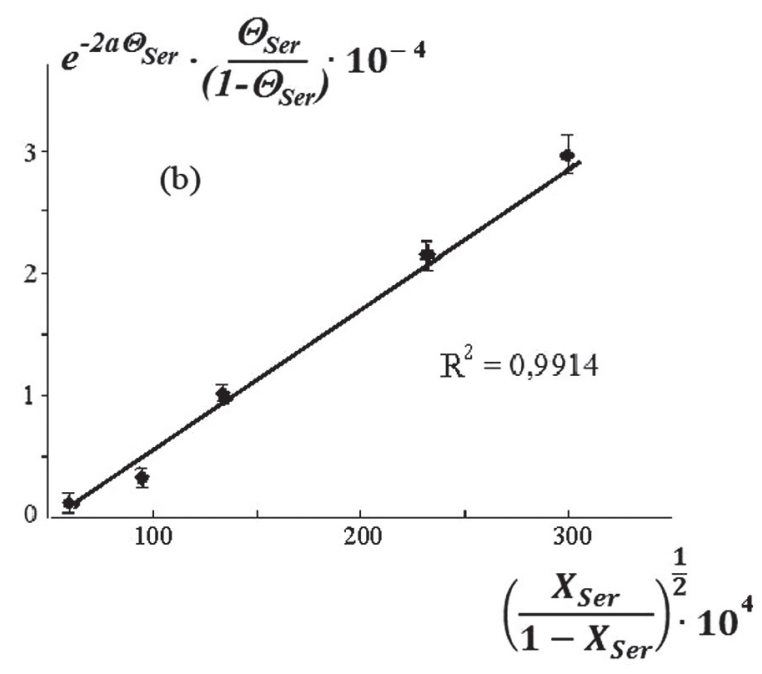

Рис. 6. Квазистационарная изотерма Темкина ( $a$ ) и Фрумкина (b) с учетом эффекта диссоциации адсорбата, полученная для процесса адсорбции аниона серина на $\mathrm{Pt}(\mathrm{Pt})$

[Fig. 6. Quasi-stationary isotherm of Temkin $(a)$ and Frumkin $(b)$, taking into account the dissociation effect of adsorbate, obtained for the adsorption of serine anion on $\mathrm{Pt}(\mathrm{Pt})$ 
аниона аминокислоты и воды задаются уравнениями:

$$
\begin{aligned}
& f_{\mathrm{Ser}^{-}}=\left.\frac{1}{R T} \frac{\partial \Delta G^{\mathrm{o}}}{\partial \Theta_{\mathrm{Ser}^{-}}}\right|_{\Theta_{\mathrm{H}_{2}}} ; \\
& f_{\mathrm{H}_{2} \mathrm{O}}=\left.\frac{1}{R T} \frac{\partial \Delta G^{\mathrm{o}}}{\partial \Theta_{\mathrm{H}_{2} \mathrm{O}}}\right|_{\Theta_{\mathrm{Ser}^{-}}} .
\end{aligned}
$$

Важно, что значения фактора $\bar{f}$, определенные из наклонов кинетических и стационарных изотерм должны совпадать.

Оказалось, что дифференциальные формы кинетических изотерм, представленных в координатах изотермы Темкина, оказались линейными (рис. 7), что позволило определить значение коэффициента $\bar{f}$, при допущении, что $\alpha=1 / 2$. При этом значения параметра $\bar{f}$, определенные по наклонам кинетической и стационарной изотерм, для данно- го электрода достаточно близки в пределах ошибки определения (табл.). Тем не менее, величина параметра $\bar{f}$, характеризующая энергетическую неоднородность $\mathrm{Pt}(\mathrm{Pt})$, почти вдвое выше, чем для гладкого Pt-электрода. Довольно высокие значения $\bar{f}$ для $\mathrm{Pt}(\mathrm{Pt})$-электрода свидетельствуют о существенном различии в энергии связи между анионом аминокислоты и атомами металла на разных участках поверхности платинированной платины. Резонно полагать, что существенная неоднородность в энергетическом плане, возможна из-за наличия значительного количества структурных дефектов на поверхности $\mathrm{Pt}(\mathrm{Pt})$.

По наклону концентрационных изотерм рассчитаны значения констант адсорбционного равновесия $K_{\text {a }}(0)$ и изменения свободной энергии Гиббса $\Delta G_{a}^{\mathrm{o}}(0)$ обменной недиссоциативной адсорб-
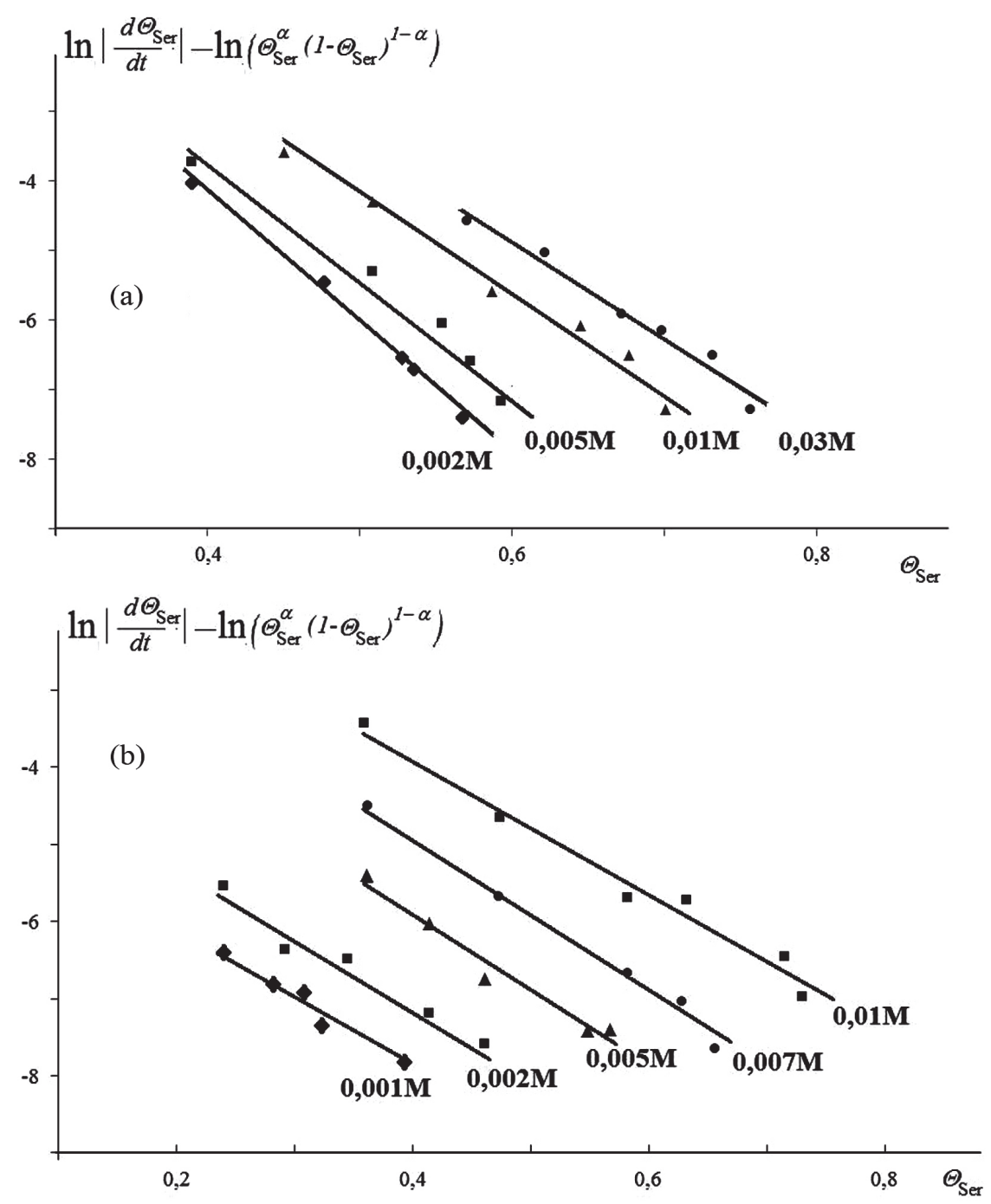

Рис. 7. Дифференциальные кинетические изотермы адсорбции Темкина для платинированного $(a)$ и гладкого $(b)$ платинового электрода

[Fig. 7. Differential kinetic of Temkin's isotherms of adsorption for $\mathrm{Pt}(\mathrm{Pt})(a)$ and smooth $(b)$ platinum electrode] 
ции аниона серина на гладкой $\mathrm{Pt}$, а также общие параметры $K_{a}^{\prime}(0)$ и $\Delta G_{a}^{\mathrm{o}^{\prime}}(0)$ - для процесса диссоциативной адсорбции аминокислоты на $\mathrm{Pt}(\mathrm{Pt})$ (табл.).

Полученные результаты, в частности, свидетельствуют о достаточно заметном хемосорбционном взаимодействии между анионом серина и поверхностью платинового электрода. Принципиально, что данное взаимодействие на платинированной платине, в отличие от гладкой, приводит к диссоциации адсорбированной частицы. Такое поведение адсорбата вряд ли является случайным. К примеру, эффект влияния структурного состояния поверхности катализатора на механизм процессов гидрогенолиза и гидрирования бензола обнаружен на рутении. Так, при увеличении степени дисперсности металла, когда средний размер частиц металла не превышает 3 nm, каталитическая активность $\mathrm{Ru}$ снижается, причем меняется природа продуктов реакции. Полагают, что подобный эффект прежде всего связан именно с различной геометрией адсорбции молекулы бензола [35-38]. Аналогичное влияние структурного состояния поверхности на природу продуктов отмечается и в работах, рассматривающих процесс дегидрирования циклогексана на поверхности Pt катализатора, представляющего собой грани монокристалла и дисперсный металл [39-45]. Наконец, различная каталитическая активность гладкого и платинированного платинового электрода при электроокислении метанола обнаружена авторами [46-47].

Таким образом, состояние поверхности гладкого и платинированного платинового электродов, несомненно, различно. Прежде всего, эти поверхности отличаются степенью энергетической неоднородности, которая на $\mathrm{Pt}(\mathrm{Pt})$ существенно выше. Резонно предположить, что и геометрия адсорбции аниона серина на гладком и платинированном платиновом электроде различна. Это может служить причиной того, что в ходе адсорбции одной и той же частицы на платинированном платиновом электроде процесс адсорбции сопровождается разрывом связей, а на гладкой Pt этого не происходит.

\section{ВЫВОДЫ}

1. Установлены основные закономерности адсорбции серина на поверхности компактного Ptи $\mathrm{Pt}(\mathrm{Pt})$-электродов в щелочной среде. Процесс адсорбции может быть описан логарифмической изотермой Темкина на энергетически равномернонеоднородной поверхности, а кинетика адсорбции подчиняется уравнению Рогинского-Зельдовича.
Таблица. Количественные характеристики процесса адсорбции аниона серина на $\mathrm{Pt}$ и $\mathrm{Pt}(\mathrm{Pt})$ электродах

[Table. Quantitative characteristics of adsorption of serine anion on $\mathrm{Pt}$ and $\mathrm{Pt}(\mathrm{Pt})$ electrodes]

\begin{tabular}{|c|c|c|c|}
\hline \multicolumn{4}{|c|}{$\begin{array}{c}\mathrm{Pt} \\
\text { (адсорбция недиссоциативная) } \\
{[\text { (adsorption is not accompanied by dissociation) }]}\end{array}$} \\
\hline $\begin{array}{l}\bar{f}_{\text {crau }} \\
{\left[\bar{f}_{\text {st }}\right]}\end{array}$ & $\begin{array}{l}\bar{f}_{\text {кинет }} \\
{\left[\bar{f}_{\text {kin }}\right]}\end{array}$ & $\ln K_{a}(0)$ & $\Delta G_{a}^{\mathrm{o}}(0)$ \\
\hline $7.5 \pm 0.9$ & $8.7 \pm 1.1$ & 14.9 & $-37.1 \mathrm{~kJ} / \mathrm{mol}$ \\
\hline \multicolumn{4}{|c|}{$\begin{array}{c}\mathrm{Pt}(\mathrm{Pt}) \\
\text { сорбция диссоциативная) }\end{array}$} \\
\hline $\begin{array}{l}\bar{f}_{\text {стац }} \\
{\left[\bar{f}_{\text {st }}\right]}\end{array}$ & $\begin{array}{l}\bar{f}_{\text {кинет }} \\
{\left[\bar{f}_{\text {kin. }}\right]}\end{array}$ & $K_{a}^{\prime}(0)$ & $\Delta G_{a}^{\mathrm{o}^{\prime}}(0)$ \\
\hline $15.5 \pm 0.8$ & $16.0 \pm 0.8$ & 16.9 & $-41.9 \mathrm{~kJ} / \mathrm{mol}$ \\
\hline
\end{tabular}

При этом на $\mathrm{Pt}(\mathrm{Pt})$-электроде адсорбция сопровождается диссоциацией серина, тогда как на гладкой платине адсорбция является недиссоциативной.

2. Значения фактора энергетической неоднородности поверхности $\bar{f}$, полученные из наклонов кинетических и стационарных изотерм, одинаковы. При этом фактор энергетической неоднородности поверхности платинированного платинового электрода практически вдвое выше аналогичного значения для гладкой Pt. Достаточно высокие значения фактора $\bar{f}$ свидетельствуют о существенной разнице в энергии связи между атомами платины и анионами серина.

3. Определены величины стандартной энергии Гиббса и констант равновесия процессов обменной недиссоциативной и диссоциативной адсорбции Ser соответственно на гладкой и платинированной платине. Полученные величины свидетельствуют о химической природе доминирующих сил взаимодействия анионов серина с поверхностью платинового электрода.

\section{КОНФЛИКТ ИНТЕРЕСОВ}

Авторы декларируют отсутствие явных и потенциальных конфликтов интересов, связанных с публикацией настоящей статьи.

\section{СПИСОК ЛИТЕРАТУРЫ}

1. Дамаскин Б. Б. Электродные проиессы в растворах органических соединений. М.: МГУ, 1985, 386 с.

2. Sobkowski J., Juzkiewics-Herbish M. Metall/Solution Interface: an Experimental Approach, Modern Aspects of Electrochemistry. № 31 / Eds. by J. O'M. Bockris, R. E. White and B. E. Conway. Plenum Press: New York, London, 1997, p. 1. 
3. Фрумкин А. Н. Избранные труды: Электродные проиессы. М.: Наука, 1987, 336 с.

4. Делахей П. Двойной слой и кинетика электродныхх проиессов. М.: Мир, 1967, 351 с.

5. Гилеади Е. Поведение промежуточных частии в электрохимическом катализе. Современные аспекты электрохимии / Под. ред. Дж. Бокриса, Б. Конуэя. М.: Мир, 1967, с. 392.

6. Electrocatalysis / Ed. by J. Lipkowski, P. N. Ross. Wiley, VCH: New York, Chichester, Weinheim, Brisbake, Singarope, Toronto, 1998. 376 p.

7. Bockris J. O. M., Shahed U. Khan M. Surface Electrochemistry: a Molecular Level Approach. Plenum Press: New York, London, 1993, 1014 p.

8. Смит А. Прикладная ИК-спектроскопия. М.: Мир, 1982. $328 \mathrm{c}$.

9. Gale J. Spectroelectrochemistry: Theory and Practice. Plenum Press: New York, 1988, p. 189.

10. Техника экспериментальных работ по электрохимии, коррозии и поверхностной обработке металлов / Под. ред. А. Т. Куна. СПб.: Химия., т. І., 1994, 560 с.

11. Lasia A. Electrochemical Impedance Spectroscopy and its Application. Modern Aspects of Electrochemistry / Eds. by B. E. Conway, J. O. M. Bockris and R. E. White. Kluwer Acad, Plenum Publ.: New York, Boston, Dordrecht, London, Moscow, 1999, p. 143.

12. Методы измерений в электрохимии / Под. ред. Э. Егер, А. Залкинд. М.: Мир, 1977. 585 с.

13. Теория хемосорбичии / Под ред. Дж. Смита. М.: Мир, 1983. 336 c.

14. Horonyi G. // J. Electroanalyt. Chem., 1975, vol. 64, iss. 1, pp. 15-19. DOI: 10.1016/0368-1874(75)80108-0

15. Huerta F., Morallon E., Cases F., Rodes A., Vazquez J. L., Aldaz A. // J. Electroanal. Chem., 1997, vol. 421, iss. 1-2, pp. 179-185. DOI: 10.1016/s00220728(96)04820-6

16. Huerta F., Morallon E., Cases F., Rodes A., Vazquez J. L., Aldaz A. // J. Electroanal. Chem., 1997, vol. 421 (1-2), pp. 155-164. DOI: 10.1016/s00220728(97)00542-1

17. Huerta F., Morallon A., Vazquez J. L, Quijada C., Berlouis L. // J. Electroanal. Chem., 2000, vol. 489, iss. 12, pp. 92-95. DOI: 10.1016/s0022-0728(00)00202-3

18. Shi-Gang Sun, Jian-Lin Yao, Qi-Hui Wu, ZhongQun Tian. // Langmuir, 2002, vol. 18, iss. 16, pp. 6274-6279. DOI: $10.1021 / \mathrm{la} 025817 \mathrm{f}$

19. Туманова Е. А., Сафронов А. Ю. // Электрохимия, 1998, т. 34, № 2, с. 170 .

20. Marangoni D. G., Smith R. S., Roscoe S. G., Marangoni D. G. // Can. J. Chem., 1989, vol. 67, iss. 5, pp. 921-926. DOI: doi.org/10.1139/v89-141

21. Ogura K., Kobayashi M., Nakayama M., Miho M. // J. Electroanal. Chem., 1998, vol. 449, iss. 1-2, pp. 101-109. DOI: 10.1016/s0022-0728(98)00015-1

22. Gu Y. J., Chen S. P., Sun S. G., Zhou Z. Y. // Langmuir, 2003, vol. 19, iss. 23, pp. 9823-9830. DOI: 10.1021/ la034758i
23. Huerta F., Morallon E., Cases F., Rodes A., Vazquez J. L., Aldaz A. // J. Electroanal. Chem., 1997, vol. 431, iss. 2, pp. 269-275. DOI: 10.1016/s0022-0728(97)00212-x

24. Huerta F., Morallon E., Vazquez J. L., Aldaz A. // J. Electroanal. Chem., 1999, vol. 475, iss. 1, pp. 38-45. DOI: 10.1016/s0022-0728(99)00337-x

25. Horanyi G. // J. Electroanal. Chem., 1991, vol. 304, iss. 1-2, pp. 211-217. DOI: 10.1016/0022-0728(91)85503-h

26. Kong De-Wen, Zhu Tian-Wei, Zeng Dong-Mei, Zhen Chun-Hua, Chen Sheng-Pei, Sun Shi-Gan. // Chem. J. Chinese Universitie, 2009, vol. 30, no. 10, p. 2040.

27. Сафонова Т. Я., Хидиров Ш. Ш., Петрий О. А. // Электрохимия, 1984, т. 20, №. 12, с. 1666.

28. Кулешова Н. Е., Введенский А. В., Бобринская Е. В. // Электрохимия, 2018, т. 54, № 7, с. 679-685. DOI: $10.1134 / \mathrm{S} 0424857018070046$

29. Фрумкин А. Н., Подловченко Б. И. // Докл. АН СССР, 1963, т. 150, № 2, с. 349 .

30. Подловченко Б. И., Иофа 3. А. // Журнал физической химии, 1964, т. 38, № 1, с. 211.

31. Дамаскин Б. Б., Петрий О. А., Цирлина Г. А. Электрохимия. М.: Химия, 2001. 623 с.

32. Дамаскин Б. Б., Петрий О. А. Введение в электрохимическую кинетику. М.: Высшая школа, 1983, 399 с.

33. Фрумкин А. Н., Багоцкий В. С., Иофа 3. А., Кабанов Б. Н. Кинетика электродньх процессов. М.: Издательство Московского университета, 1952, 319 с.

34. Бобринская Е. В., Введенский А. В., Карташова Т. В., Кращенко Т. Г. // Коррозия: материаль, защита, 2013 , № 8, c. 1 .

35. Брагин О. В., Либерман А. Л. // Успехи химии, 1970 , № 12 , c. 2122.

36. Anderson I. R., Macdonald R. I., Shimoyama Y. // J. Catalysis, 1971, vol. 20, № 2, p. 147. DOI: 10.1016/00219517(71)90076-5

37. Levitskii I. L, Minachev Kh. M. In: Mechanisms of Hydrocarbon Reactions. 1973, Budapest, Academiai Kiado, 1975, Preprint, № 15, p. 81.

38. Anderson J. R., Baker B. G. Chemisorption and Reactions on Metallic Films. London, New-York, Acad. Press, 1971, p. 63.

39. Брагин О. В. Преображенский А. В., Либерман А. Л., Казанский Б. А. // Кинетика и катализ, 1975, т. 16, № 2, с. 472 .

40. Maire G., Corolleur C., Juttard D., Gault F. G. // J. Catalysis, 1971, vol. 21, iss. 2, pp. 250-253. DOI: 10.1016/0021-9517(71)90143-6

41. Corolleur C., Corolleur S., Gault F. G. // J. Catalysis, 1972, vol. 24, iss. 3, pp. 385-400. DOI: 10.1016/00219517(72)90123-6

42. Paal Z., Tetenyi P. // Acta. Chim. Acad. Sci. Hung., 1972, vol. 72, № 3, p. 277.

43. Barron Y., Maire G., Muller J. M., Gault F. G. // J. Catalysis, 1966, vol. 5, iss. 3, pp. 428-445. DOI: 10.1016/ s0021-9517(66)80062-3

44. Muller J. M., Gault F. G. // J. Catalysis, 1972, vol. 24, iss. 2, pp. 361-364. DOI: 10.1016/0021-9517(72)90083-8 
45. Contreras A. M., Grunes J., Yan X.-M., Liddle A., Somorjai G. A. // Topics in Catalysis. 2006, vol. 39, iss. 34, pp. 123-129. DOI: 10.1007/s11244-006-0047-0
46. Хазова А. М., Васильев Ю. Б., Багоцкий В. С. // Электрохимия, 1967, т. 3, с. 1020.

47. Подловченко Б. И., Петухова Р. П. // Электрохимия, 1972, т. 8, № 6, с. 899 .

UDC 544.65:544.4+544.653.23:547.1-32-304.2

DOI: $10.17308 / \mathrm{kcmf} .2019 .21 / 718$

Received 19.12.2018

Accepted 15.02.2019

\title{
THE EFFECT OF THE STRUCTURAL AND MORPHOLOGICAL PROPERTIES OF THE SURFACE OF PLATINUM ON THE KINETIC AND THERMODYNAMIC CHARACTERISTICS OF THE SERINE ANION ADSORPTION PROCESS
}

\author{
(C) 2019 N. E. Kuleshova, A. V. Vvedenskii*, E. V. Bobrinskaya, E. B. Rychkova \\ Voronezh State University \\ 1, Universitetskaya pl., 394018 Voronezh, Russian Federation
}

\begin{abstract}
Purpose. The purpose of this paper is to establish the influence of the differences in the structural and morphological properties of the surface of $\mathrm{Pt}$ and $\mathrm{Pt}(\mathrm{Pt})$ electrodes (roughness, in particular) on the basic principles and thermodynamic characteristics of the adsorption process of serine anion.

Methods and methodology. Kinetic isotherms of adsorption of serine anion on a smooth and platinized platinum electrode were obtained by electrooxidation in the adsorbed layer (charging curves). The true surface area of the corresponding platinum electrode was calculated using the charge in the desorption region of atomic hydrogen. The adsorption potential was selected following the results of linear voltammetry. The criterion for selection was the absence of the oxidation/reduction process of the amino acid anion. It was found that the process of adsorption of serine anion on platinum can be described by the logarithmic Temkin isotherm on an energetically uniformly inhomogeneous surface, and the adsorption kinetics can be described by the Roginsky-Zeldovich equation. Adsorption on a $\mathrm{Pt}(\mathrm{Pt})$ electrode is accompanied by serine dissociation, whereas adsorption on smooth platinum is non-dissociative.

Results. The calculated energetically inhomogeneity factor of the platinized platinum electrode surface is almost twice as high as that of a smooth Pt surface. Such high values indicate a significant difference in the binding energy between platinum atoms and anions of serine.

Conclusions. The standard Gibbs energy and the equilibrium constants were calculated for the processes of exchange dissociative and non-dissociative adsorption of serine on a smooth surface and platinized Pt respectively. The obtained values indicate the chemical nature of the dominant forces of interaction between anions of serine and the surface of the platinum electrode.
\end{abstract}

Keywords: serine, amino acid, platinum, adsorption, isotherm.

\section{CONFLICT OF INTEREST}

The authors declare the absence of obvious and potential conflicts of interest related to the publication of this article.

\section{REFERENCES}

1. Damaskin B., Petrii A. O., and Batrakar V. Adsorption of Organic Compounds on Electrodes. Plenum Press, New York, 1973.

2. Sobkowski J., Juzkiewics-Herbish M. Metall/Solution Interface: an Experimental Approach, Modern Aspects of

$\triangle$ Vvedenskii Alexander V., e-mail: alvved@chem.vsu.ru
Electrochemistry, no. 31. Eds. by J. O'M. Bockris, R. E. White and B. E. Conway. Plenum Press, New York, London, 1997, p. 1.

3. Frumkin A. N. Isbrannie trudi: Electrodnie processi, [Selected Works: Electrode Processes]. Moscow, Nauka Publ., 1987. 336 p. (in Russ.)

4. Delahey P. Dvoinoi sloi i kinetika elektrodnih processov, [Double Layer and Kinetics of Electrode Processes]. Moscow, Mir Publ., 1967, 351 p. (in Russ.)

5. Gileadi E. and Conway B. in: Modern Aspects of Electrochemistry, no. 3 Eds. by J. O’M. Bockris and B. Conway. Butterworths, London, 1964. 
6. Electrocatalysis. Ed. by J. Lipkowski, P. N. Ross. Wiley, VCH, New York, Chichester, Weinheim, Brisbake, Singarope, Toronto, 1998, $376 \mathrm{p}$.

7. Bockris J. O. M., Shahed U. Khan M. Surface Electrochemistry: a Molecular Level Approach. Plenum Press, New York, London, 1993, 1014 p.

8. Applied Infrared Spectroscopy. By A. Lee Smith. Wiley, Chichester, 1979.

9. Gale J. Spectroelectrochemistry: Theory and Practice. Plenum Press, New York, 1988, p. 189.

10. Tehnika eksperimentalnih rabot po electrohimii, korrosii I poverhnostnoi obrabotke metallov [Technique of Experimental Work on Electrochemistry, Corrosion and Surface Treatment of Metals]. Ed. by A. T. Kuna. Saint Petersburg, Khimiya Publ., vol. I., 1994, 560 p. (in Russ.)

11. Lasia A. Electrochemical Impedance Spectroscopy and its Application. Modern Aspects of Electrochemistry. Eds. by B. E. Conway, J. O.' M. Bockris and R. E. White. Kluwer Acad, Plenum Publ., New York, Boston, Dordrecht, London, Moscow, 1999, p. 143.

12. Metodi ismerenii $v$ elektrohimii [Measurement Methods in Electrochemistry]. Ed. by E. Eger, A. Zalkind. Moscow, Mir Publ., 1997, 585 p. (in Russ.)

13. Theory of Chemisorption. Ed. by J. Smith. Berlin, Springer, 1980, $240 \mathrm{p}$.

14. Horønyi G. J. Electroanalyt. Chem., 1975, vol. 64, iss. 1, pp. 15-19. DOI: 10.1016/0368-1874(75)80108-0

15. Huerta F., Morallon E., Cases F., Rodes A., Vazquez J. L., Aldaz A. // J. Electroanal. Chem., 1997, vol. 421, iss. 1-2, pp. 179-185. DOI: $10.1016 / \mathrm{s} 0022-$ 0728(96)04820-6

16. Huerta F., Morallon E., Cases F., Rodes A., Vazquez J. L., Aldaz A. // J. Electroanal. Chem., 1997, vol. 421 (1-2), pp. 155-164. DOI: 10.1016/s00220728(97)00542-1

17. Huerta F., Morallon A., Vazquez J. L, Quijada C., Berlouis L. J. Electroanal. Chem., 2000, vol. 489, iss. 1-2, pp. 92-95. DOI: 10.1016/s0022-0728(00)00202-3

18. Shi-Gang Sun, Jian-Lin Yao, Qi-Hui Wu, ZhongQun Tian. Langmuir, 2002, vol. 18, iss. 16, pp. 6274-6279. DOI: $10.1021 / \mathrm{la} 025817 \mathrm{f}$

19. Tumanova E. A., Safonov A. Yu. Elektrokhimiya [Russian Journal of Electrochemistry], 1998, vol. 34, iss. 2, p. 153. (in Russ.)

20. Marangoni D. G., Smith R. S., Roscoe S. G., Marangoni D. G. Can. J. Chem., 1989, vol. 67, iss. 5, pp. 921926. DOI: doi.org/10.1139/v89-141

21. Ogura K., Kobayashi M., Nakayama M., Miho M. J. Electroanal. Chem., 1998, vol. 449, iss. 1-2, pp. 101-109. DOI: 10.1016/s0022-0728(98)00015-1

22. Gu Y. J., Chen S. P., Sun S. G., Zhou Z. Y. Langmuir, 2003, vol. 19, iss. 23, pp. 9823-9830. DOI: 10.1021/ la034758i

23. Huerta F., Morallon E., Cases F., Rodes A., Vazquez J. L., Aldaz A. J. Electroanal. Chem., 1997, vol. 431, iss. 2, pp. 269-275. DOI: 10.1016/s00220728(97)00212-x
24. Huerta F., Morallon E., Vazquez J. L., Aldaz A. J. Electroanal. Chem., 1999, vol. 475, iss. 1, pp. 38-45. DOI: 10.1016/s0022-0728(99)00337-x

25. Horanyi G. J. Electroanal. Chem., 1991, vol. 304, iss. 1-2, pp. 211-217. DOI: 10.1016/0022-0728(91)85503$\mathrm{h}$

26. Kong De-Wen, Zhu Tian-Wei, Zeng Dong-Mei, Zhen Chun-Hua, Chen Sheng-Pei, Sun Shi-Gan. Chem. J. Chinese Universitie, 2009, vol. 30, no. 10, p. 2040.

27. Safonova T. Y., Hidirov Sh. Sh., Petrii O. A. Elektrokhimiya [Russian Journal of Electrochemistry], 1984, vol. 20, iss. 12, p. 1666. (in Russ.)

28. Kuleshova N. E., Vvedenskyi A. V., Bobrinskaya E. V. Electrokchimiya [Russian Journal of Electrochemistry], 2018, vol. 54, iss. 7, pp. 592-597. DOI: 10.1134/ S1023193518070042

29. Frumkin A. N., Podlovchenko B. I. Dokl. AN SSSR, 1963, vol. 150, iss. 2, p. 349. (in Russ.)

30. Podlovchenko B. I., Iofa Z. A. Journal fisicheskoi himii [Russian Journal of Physical Chemistry A], 1964, vol. 38, no. 1, p. 211. (in Russ.)

31. Damaskin B. B., Petrii O. A., Tsyrlina G. A. Electrokhimiya [Electrochemistry]. Moscow, Khimiya Publ., 2001, 623 p. (in Russ.)

32. Damaskin B. B., Petrii O. A., Vvedenie v electrokhimiceskyu kinetiku [Introduction to Electrochemical Kinetics]. Moscow, Vyshaya Shkola Publ., 1983, 399 p. (in Russ.)

33. Frumkin A. N., Bagotskii V. S., Iofa Z. A. Kabanov B. N. Kinetika elektrodnyh processov [Kinetics of Electrode Processes]. Moscow, Izdat. Moskovs.Universiteta Publ., 1952, 319 p. (in Russ.)

34. Bobrinskaya E. V., Vvedenskyi A. V., Kartashova T. V., Krashenko T. G. Korrosia: materialy i zashita [Corrosion: Materials, Protection], 2013, no. 8, pp. 1-8. (in Russ.)

35. Bragin O. V., Liberman A. L. Russian Chemical Reviews, 1970, vol. 39, no. 12, p. 1017. DOI: 10.1070/RC 1970v039n12ABEH002315

36. Anderson I. R., Macdonald R. I., Shimoyama Y. J. Catalysis, 1971, vol. 20, № 2, p. 147. DOI: 10.1016/00219517(71)90076-5

37. Levitskii I. L, Minachev Kh. M. In: Mechanisms of Hydrocarbon Reactions. 1973, Budapest, Academiai Kiado, 1975, Preprint, no. 15, p. 81.

38. Anderson J. R., Baker B. G. Chemisorption and Reactions on Metallic Films. London, New-York. Acad. Press, 1971, p. 63.

39. Bragin O. V., Preobrazenskii A. V., Liberman A. L., Kazanskii B. A. Kinetica i katalys [Kinetics and Catalysis], 1975, vol. 16, no. 2, p. 472. (in Russ.)

40. Maire G., Corolleur C., Juttard D., Gault F. G. J. Catalysis, 1971, vol. 21, iss. 2, pp. 250-253. DOI: 10.1016/0021-9517(71)90143-6

41. Corolleur C., Corolleur S., Gault F. G. J. Catalysis, 1972, vol. 24, iss. 3, pp. 385-400. DOI: 10.1016/00219517(72)90123-6 
42. Paal Z., Tetenyi P. Acta. Chim. Acad. Sci. Hung., 1972, vol. 72, no. 3, p. 277.

43. Barron Y., Maire G., Muller J. M., Gault F. G. $J$. Catalysis, 1966, vol. 5, iss. 3, pp. 428-445. DOI: 10.1016/ s0021-9517(66)80062-3

44. Muller J. M., Gault F. G. J. Catalysis, 1972, vol. 24, iss. 2, pp. 361-364. DOI: 10.1016/0021-9517(72)90083-8
45. Contreras A. M., Grunes J., Yan X.-M., Liddle A., Somorjai G. A. Topics in Catalysis. 2006, vol. 39, iss. 3-4, pp. 123-129. DOI: 10.1007/s11244-006-0047-0

46. Khazova A. M., Vasil'ev U. B., Bagotskii V. S. Soviet Electrochemistry, 1967, vol. 3, no. 7, p. 1020. (in Russ.)

47. Podlovchenko B. I., Petuhova R. P. Soviet Electro-chemistry, 1972, vol. 8, no. 6, p. 899. (in Russ.)
Кулешова Надежда Евгеньевна - аспирант кафедры физической химии, Воронежский государственный университет, Воронеж, Российская Федерация; e-mail kuleshova_nadya28@mail.ru. ORCID iD 0000-0002-0878-2663.

Введенский Александр Викторович - д. х. н., профессор, зав. кафедрой физической химии, Воронежский государственный университет, Воронеж, Российская Федерация; e-mail: alvved@chem. vsu.ru. ORCID iD 0000-003-2210-5543.

Бобринская Елена Валерьевна - к. х. н., доцент кафедры физической химии, Воронежский государственный университет, Воронеж, Российская Федерация; e-mail elena173.68@mail.ru. ORCID iD 0000-0001-7123-4224.

Рычкова Елена Борисовна - магистрант 2 года обучения, химический факультет Воронежский государственный университет, Воронеж, Российская Федерация; ORCID iD 0000-0002-4957-6196.
Kuleshova Nadezhda E. - postgraduate student of the Physical Chemistry Department, Voronezh State University, Voronezh, Russian Federation; e-mail kuleshova_nadya28@mail.ru. ORCID iD 0000-00020878-2663.

Vvedenskii Alexander V.- Dr. Sci. (Chem.), Full Professor, Head of the Physical Chemistry Department, Voronezh State University, Voronezh, Russian Federation; e-mail: alvved@chem.vsu.ru. ORCID iD 0000-0003-2210-5543

Bobrinskaya Elena V. - Cand. Sci. (Chem.), Associate Professor of the Physical Chemistry Department, Voronezh State University, Voronezh, Russian Federation; e-mail elena173.68@mail.ru. ORCID iD 0000-0001-7123-4224.

Rychkova Elena B. $-2^{\text {rd }}$ year Master's Degree of the Physical Chemistry Department, Voronezh State University, Voronezh, Russian Federation; ORCID iD 0000-0002-4957-6196. 